Document downloaded from:

http://hdl.handle.net/10251/65899

This paper must be cited as:

Giner Sanz, JJ.; Ortega Navarro, EM.; Pérez-Herranz, V. (2015). Montecarlo based quantitative Kramers-Kronig test for PEMFC impedance spectrum validation. International Journal of Hydrogen Energy. 40(34):11279-11293. doi:10.1016/j.jjhydene.2015.03.135.

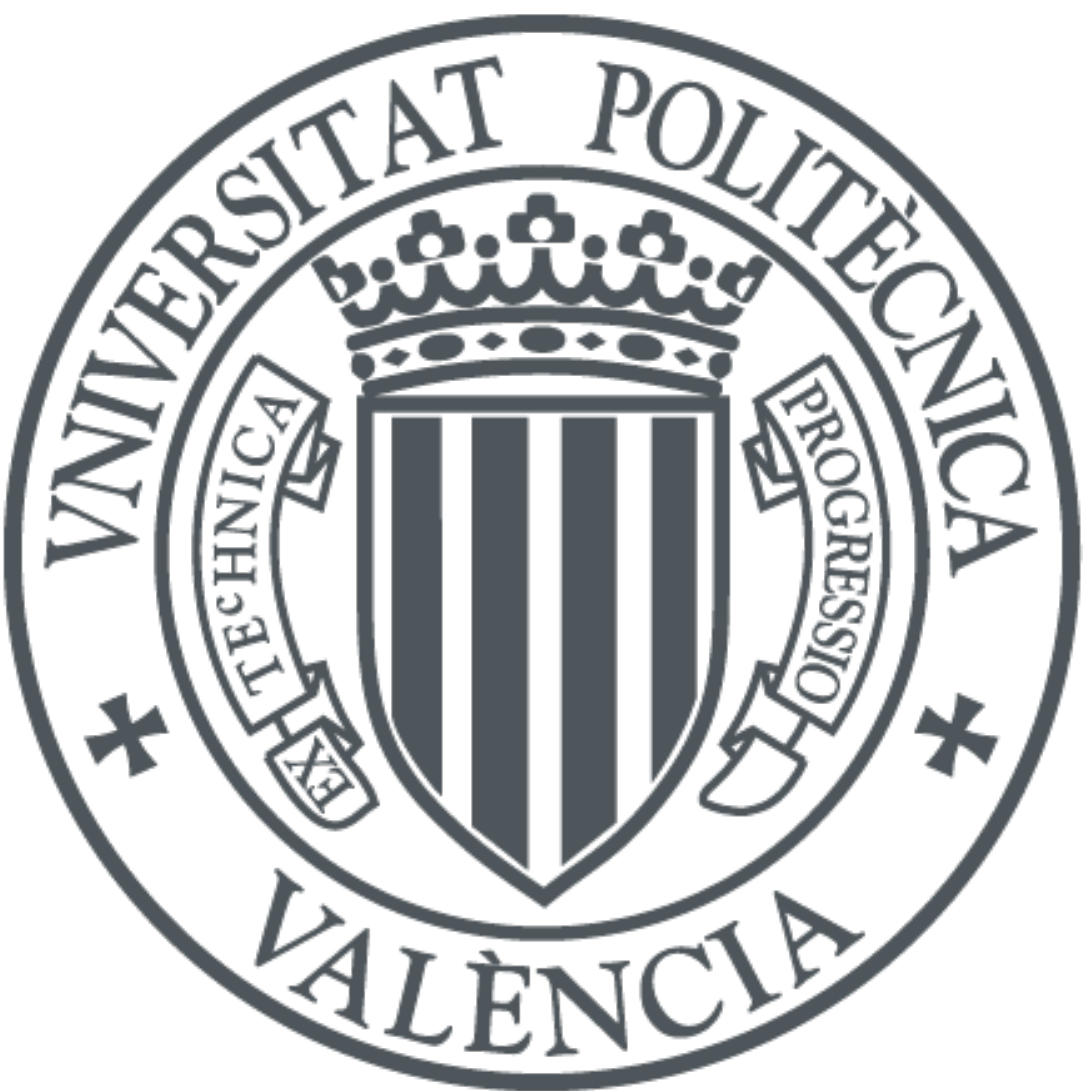

The final publication is available at

http://dx.doi.org/10.1016/j.ijhydene.2015.03.135

Copyright Elsevier

Additional Information 


\title{
Montecarlo based quantitative Kramers-Kronig test for PEMFC impedance spectrum validation
}

\author{
J. J. Giner-Sanz, E. M. Ortega, V. Pérez-Herranz* \\ IEC group, Depto. Ingeniería Química y Nuclear, Universitat Politècnica de València \\ Camino de Vera S/N, 46022 Valencia, Spain \\ *Corresponding author. Tel.: +34-96-3877632; fax: +34-96-3877639; \\ E-mail address: vperez@iqn.upv.es (V.Pérez-Herranz)
}

Keywords: Electrochemical Impedance Spectroscopy, Kramers-Kronig, validation, Montecarlo algorithm, PEMFC.

\section{Abstract}

Electrochemical Impedance Spectroscopy (EIS) is a very powerful tool to study the behaviour of electrochemical systems. At present, it is widely used in the fuel cell field in order to study challenging cutting edge issues as membrane drying or gas diffusion layer flooding amongst others. The proper analysis of impedance data requires the fulfilment of four fundamental conditions: causality, linearity, stability and finiteness. The non compliance with any of these conditions may lead to biased, or even misguided, conclusions. Therefore it is critical to verify the compliance of these conditions before accepting any analysis performed on an experimental spectrum. This is even more important in a fuel cell experimental spectrum analysis, since fuel cells are markedly non stationary systems. The aim of this work is to establish an impedance spectrum quantitative validation technique to validate the whole experimental spectrum and to identify the individual points within a spectrum that do not comply any of the four conditions, in order to remove these inconsistent points from the analysis. The designed validation method consists in a Kramers-Kronig (KK) validation test, by equivalent electrical circuit fitting, coupled with a Montecarlo error propagation method. In a first step, the experimental spectrum is fitted to a particular electrical equivalent circuit, which satisfies the KK relations. Then, in a second step, a statistical Montecarlo method is used in order to propagate the model fitting parameter uncertainty through the model. Using this approach, a consistency region is built for a given confidence level: the experimental points inside this region are considered consistent for the given confidence level, whereas the outside points are rejected. The method was used on PEMFC experimental impedance spectra; and it successfully managed to identify inconsistent points, associated to no stationarities. 


\section{Introduction}

In current days, electrochemical impedance spectroscopy (EIS) has gained significant relevance in the fuel cell (FC) field, since this electrochemical measurement technique allows to obtain information on the fuel cell internal state and on its electrochemical behaviour [1-2]. This technique provides detailed information on the conductivity of the membrane, on the electrochemical electrode processes and on the intrinsic losses of the system [3-5]. All these data are crucial in order to tackle some of the most challenging actual issues of fuel cells, such as membrane drying and gas diffusion layer flooding [6-7]. Therefore, EIS has widely been applied for membrane electrode assembly optimization [8-13]; operation conditions optimization [14-17]; control [18] and diagnosis [19-20]. The technique has been applied both, to fuel cell single cells [20$24]$ and to fuel cell stacks $[19,25]$.

The impedance of a given system for angular frequency $\omega$ is defined by complex Ohm's law [26]:

$$
Z(\omega)=\frac{\mathcal{F}[U(t)]}{\mathcal{F}[I(t)]}
$$

Where $\mathcal{F}$ represents the Fourier transform operator. The impedance concept is a generalization of the DC electric resistance concept: it quantifies not only the electric resistance of the system (amplitude relation between the current and the voltage signals); but it also quantifies the time offset of both signals.

The generalized Ohm's law, and thus the impedance concept, are valid only if the hypothesis of linearity, causality, finite range and stability are met [27]. If any of these conditions is not fulfilled the obtained results may be misleading and unusable to extract proper conclusions on the system. Therefore it is crucial to verify that the experimental impedance spectrum satisfies the four conditions, before starting the analysis itself [28]. Experimental spectrum validation is even more important for fuel cell systems, since these systems are highly non stationary, and generally present potential time drifts [29].

Firstly, the linearity of the system is essential for the no-generation of harmonics, which would distort the obtained EIS spectra [30]. However, the electrochemical systems are in general highly nonlinear systems since they are governed by ButtlerVolmer's equation. In these cases, the linearity condition is achieved by applying a perturbation of small enough amplitude [20]. 
Secondly, the causality condition implies that the system's response is a direct consequence of the perturbation; and therefore, the response does not precede the disturbance [31]. The finite range condition implies that the system's impedance takes a finite value in the whole frequency range [28]. In practice, this condition is always met, since physical systems are finite systems; and therefore, the finite value condition is not a critical condition [26].

Finally, a system meets the stability condition if it operates in a single operation point during the measurement of the whole EIS spectrum [32]. Due to stochastic processes, as flooding and drying; to aging processes; and to variations of the input parameters, fuel cell systems are generally highly non stable systems [19]. In consequence, the stability condition is the most critical condition for EIS measurements in FC systems.

The main purpose of this work is to establish an experimental impedance spectrum quantitative validation technique. The validation test is not just meant to validate the experimental spectrum or not (verify if the four conditions are satisfied by the whole spectrum); but it also has to distinguish the individual spectrum points that do not meet any of the four conditions. The removal of the inconsistent points allows using the rest of the spectrum for the analysis, thus reducing the number of spectra that have to be repeated due to the presence of a little amount of inconsistent points. The validation test was experimentally validated by measuring the EIS spectra of a single cell of a commercial PEMFC stack, in operation conditions in which the system was stable and in operation conditions in which it was unstable. This work focuses on the validation of the developed method for no stability detection; since, as it has been explained previously, the stability condition is the most critical one in FC systems. 


\section{Methodology}

\subsection{Kramers-Kronig relations}

The Kramers-Kronig (KK) relations are integral equations relating the real part and the imaginary part of complex quantities that meet the conditions of causality, linearity, finite value and stability. They were first obtained by Kramers [33] and Kronig [34] in the field of optics; and they were extrapolated to the electrical impedances field by Bode [35]. They come from the Cauchy theorem that introduces the definition of a causal system. They establish that:

$$
\begin{gathered}
Z^{\prime \prime}(\omega)=\left(\frac{2 \omega}{\pi}\right) \cdot \int_{0}^{+\infty} \frac{Z^{\prime}(x)-Z^{\prime}(\omega)}{x^{2}-\omega^{2}} \cdot d x \\
Z^{\prime}(\omega)=\lim _{\omega \rightarrow+\infty}\left[Z^{\prime}(\omega)\right]+\left(\frac{2}{\pi}\right) \cdot \int_{0}^{+\infty} \frac{x \cdot Z^{\prime \prime}(x)-\omega \cdot Z^{\prime \prime}(\omega)}{x^{2}-\omega^{2}} \cdot d x
\end{gathered}
$$

Where $Z^{\prime}$ and $Z^{\prime \prime}$ denote respectively the real and the imaginary part of the impedance; $\omega$ stands for the angular frequency, $2 \pi f$.

It should be kept in mind that these relations are purely mathematical relations, and therefore, they do not reflect any actual physical condition of the studied system.

The KK relations are a powerful tool for impedance spectrum consistency verification [36], since a consistent spectrum (a spectrum that verifies the four conditions) satisfies KK relations; and vice versa, a spectrum that satisfies KK relations verifies the four conditions, and therefore is consistent. So evaluating if the experimental spectrum satisfies KK relations is a good consistency test.

Even if in theory, the KK relations are able to detect non-compliance with any of the four conditions; in practice, they are much more susceptible to instabilities that to nonlinearities [29]. For example, in the system investigated by Urquidi-Macdonald [37] it was observed that $\mathrm{KK}$ relations were virtually insensitive to nonlinearities: KK relations were satisfied even when the system did not fulfil the linearity condition. Recently it has been shown that the Kramers-Kronig relations are only sensitive to nonlinearities if at least a part of the spectrum has been measured for frequencies above a transition frequency, which depends on the system [38]. On the other hand, it has been widely observed that the $\mathrm{KK}$ relations are very sensitive to the non- 
compliance of the stability condition: even very slightly unstable systems do not satisfy KK relations [39].

In bibliography, KK relations have been applied by direct integration of the KK equations; by experimental observation of linearity, finiteness and stationarity; and by fitting to an electrical equivalent circuit that satisfies Kramers-Kronig relations [36].

On the one hand, direct integration of KK equations allows to calculate one of the impedance components (real part o imaginary part) from the other one. The calculated component can then be compared with the experimental one; and conclusions about the compliance of the $\mathrm{KK}$ relations can be extracted. The main limitation of this method is that KK relations direct integration requires data in the frequency range from 0 to infinity [40]; and the data obtained in practice only cover a finite frequency range. Thus, extrapolation of the data is required. Several extrapolation methods have been used in literature: the Kendig method [41-42]; the Macdonald method [43]; the Haili method [44] and the Esteban-Orazem method [40]. However, any of these methods can be used if the measured frequency range is too narrow; or if the maximum imaginary component point has not been obtained experimentally [36].

On the other hand, the experimental validation of KK relations consists in verifying experimentally that the conditions of causality, linearity and stability are satisfied: if so, it can be deduced that the KK are also satisfied. The hypotheses of causality and linearity can be experimentally verified analysing the response of the system in the frequency domain [45-46]: if these hypotheses are satisfied the non fundamental harmonic content in the response signal should be neglectable [26]. The hypothesis of stability can be experimentally verified by replication of the spectra measurement [28].

Finally, if a system can be fit to an equivalent circuit that verifies KK relations, then the system also satisfies these relations [47]. Circuits consisting in passive and distributed elements satisfy KK relations [48]. Combining both ideas it can be deduced that if the experimental EIS spectrum (which has to be validated) can be fitted to an equivalent circuit formed by passive and distributed elements, then the experimental spectrum is consistent with KK relations.

The equivalent circuit fitting methodology has the great advantage of not requiring the evaluation of the integral equations in an infinite frequency range. Therefore it does not require the use of extrapolation algorithms; which, as mentioned previously, introduce errors in the analysis and have a very limited applicability. However, it presents a limitation: the equivalent circuit and the parameter initialization have to be carefully selected; since a bad selection can lead to the test failure. 


\subsection{Overall methodology selection}

Considering the advantages and the drawbacks of each one of both methods, it was decided to select the equivalent circuit fitting method for this work. The experimental spectra were fitted to an equivalent electrical circuit that satisfies KK relations in order to validate the experimental spectra:

- If a spectrum is fit by the equivalent circuit, then it is consistent with KK relations: it can be considered valid; and can be used for further analysis;

- If a spectrum is not fit by the equivalent circuit, then it is inconsistent with KK relations: it cannot be considered valid; and if used for further analysis, it could lead to slanted or erroneous conclusions.

An issue arises from this methodology: the definition of "good" fit. The definition of the acceptance criteria of the test (the definition of "good" and "bad" fits) is not trivial and depends on the studied system [36].

In order to overcome the issue of the acceptance criteria definition, a quantitative method based on a Montecarlo algorithm was used. This quantitative method will set the acceptance criteria for each point of the experimental spectrum, for a given confidence level. Moreover, since this method sets different acceptance criteria for each point, it will detect inconsistent points individually rather than working with the spectrum as a whole. Therefore, the method will allow distinguishing which points of the experimental spectrum are consistent and which ones are inconsistent with the KK relations.

\subsection{Circuit fitting}

As introduced previously, in the present work the equivalent circuit fitting method was selected. The first step consists in the selection of the equivalent circuit to which the experimental spectra are going to be fit. On the one hand, Bastidas and co-workers suggest using Voight's circuit [49]. Voight's circuit, given in figure 1 , is a $R(R C)_{n}$ circuit: it is composed by $n$ parallel $R C$ subcircuits in series with an individual resistance. The main advantages of this circuit for this work are:

- Firstly, it is a very versatile circuit that can fit properly a wide range of impedance spectra, just by modifying the number of considered sub circuits, $n$. It can even fit inductive parts with negative resistance and capacitance values. Since this is only a validation technique, these negative values can be accepted for performing the spectrum validation, even if they don't have any physical meaning for the studied system [50]. 
- Secondly, this circuit satisfies the Kramers-Kronig relations, and therefore if the experimental spectrum can be fitted by this circuit, then the experimental spectrum satisfies Kramers-Kronig relations.

On the other hand, Boukamp suggests using Boukamp's circuit [48], which consists in a Voight's circuit in series with a capacitor and an inductor. It is a $R(R C)_{n} C L$ circuit. This circuit has the same advantages than Voight's circuit; but it has two supplementary parameters. Since Voight's circuit is able to model inductive parts, as it has been explained above, both circuits are equivalent for this work's purposes. In consequence, Voight's circuit was selected in this work, since it involves less parameters.

Using the impedance expressions for resistors and capacitors, and the impedance combination rules [51], the following expressions were obtained for the $R(R C)_{n}$ circuit:

$$
\begin{gathered}
Z_{R(R C)_{n}}^{\prime}(\omega)=R_{1}+\sum_{i=1}^{n}\left(\frac{R_{R C_{i}}}{\left(R_{R C_{i}} \cdot C_{R C_{i}} \cdot \omega\right)^{2}+1}\right) \\
Z^{\prime \prime}{ }_{R(R C)_{n}}(\omega)=-\sum_{i=1}^{n}\left(\frac{R_{R C_{i}}{ }^{2} \cdot C_{R C_{i}} \cdot \omega}{\left(R_{R C_{i}} \cdot C_{R C_{i}} \cdot \omega\right)^{2}+1}\right)
\end{gathered}
$$

Where $Z^{\prime}{ }_{R(R C)_{n}}$ and $Z^{\prime \prime}{ }_{R(R C)_{n}}$ denote respectively the real and the imaginary part of the impedance of the $R(R C)_{n}$ circuit; $R_{1}$ represents the individual resistance; and finally, $R_{R C_{i}}$ and $C_{R C_{i}}$ are the resistance and the capacitance of the $i$-th $R C$ subcircuit. The vector of parameters associated with the $R(R C)_{n}$ circuit, $\vec{a}_{R(R C)_{n}}$, is a $2 n+1$ dimensional vector, defined as:

$$
\vec{a}_{R(R C)_{n}}=\left(\begin{array}{c}
R_{1} \\
R_{R C_{1}} \\
C_{R C_{1}} \\
\vdots \\
R_{R C_{n}} \\
C_{R C_{n}}
\end{array}\right)_{2 n+1}
$$

Actually, a large number of programs are able to perform equivalent circuit fitting to

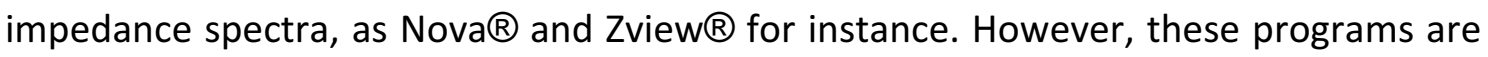
not useful in this case; since the considered circuit is a circuit with a variable number of elements (the number varies from one experimental spectrum to another), which can need a large number of elements to fit the experimental spectrum. The use of these 
programs would require modifying the fitting circuit manually after each iteration, which is infeasible in this work's context. This is the reason why in this work a Labview ${ }^{\circledR}$ program was developed in order to perform the fitting of experimental spectra to Voight's circuit.

The fitting process consists in determining the vector of parameters, $\vec{a}_{R(R C)_{n}}$, that minimizes the fitting error (difference between each one of the experimental points and the associated predicted-by-the-model point). In order to quantify the fitting error a goodness of fit parameter must be defined. In the EIS field, the most used goodness of fit parameter is the weighted sum of quadratic errors [28], $\chi^{2}$. This parameter is defined as:

$$
\chi^{2}=\sum_{i=1}^{N_{p}}\left[\alpha_{i} \cdot\left(Z^{\prime} \exp \left(\omega_{i}\right)-Z_{R(R C)_{n}}^{\prime}\left(\omega_{i}\right)\right)^{2}+\beta_{i} \cdot\left(Z^{\prime \prime}{ }_{\exp }\left(\omega_{i}\right)-Z_{R(R C)_{n}}^{\prime \prime}\left(\omega_{i}\right)\right)^{2}\right]
$$

Where $N_{p}$ denotes the number of experimental points. Subscript exp refers to experimental points; whereas, subscript $R(R C)_{n}$ refers to model calculated points using equations (4) and (5). Finally, $\alpha_{i}$ and $\beta_{i}$ are the weight of the real and the imaginary part of point $i$.

The optimum weighing strategy is to weight each component of each experimental point inversely to the uncertainty associated to it [26]: experimental data with low uncertainties will be given high weights in the fitting; whereas, experimental data with big uncertainties will be given low weights, preventing the noise in the fitting that these points would introduce if given a big weight. Thus the optimum weights are given by:

$$
\begin{aligned}
& \alpha_{i}=\frac{1}{\left|\sigma_{Z^{\prime}\left(\omega_{i}\right)}\right|^{2}} \\
& \beta_{i}=\frac{1}{\left|\sigma_{Z^{\prime \prime}\left(\omega_{i}\right)}\right|^{2}}
\end{aligned}
$$

Where $\sigma_{Z^{\prime}\left(\omega_{i}\right)}$ and $\sigma_{Z^{\prime \prime}\left(\omega_{i}\right)}$ denote the uncertianty of the real and the imaginary part associated to the $i$-th point.

An hypothesis that can generally be applied is that the relative errors associated to measurements are constant [52]. Thus, the absolute error (the uncertainty) is proportional to the impedance modulus: 


$$
\begin{aligned}
\sigma_{Z^{\prime}\left(\omega_{i}\right)} & \propto\left|Z_{\text {exp }}\left(\omega_{i}\right)\right| \\
\sigma_{Z^{\prime \prime}\left(\omega_{i}\right)} & \propto\left|Z_{\text {exp }}\left(\omega_{i}\right)\right|
\end{aligned}
$$

The value of the proportionality constant has no effect on the fitting [53]: therefore, it was considered as the unity. Introducing equations (10) and (11) in equations (8) and (9):

$$
\alpha_{i}=\beta_{i}=\frac{1}{\left|Z_{\exp }\left(\omega_{i}\right)\right|^{2}}
$$

In this work, the weights (real part and imaginary part weights) of each of the experimental point were determined using equation (12). Once each point has been weighted, the parameter $\chi^{2}$ can be calculated for a given vector of parameters. The following step is to proceed to the fitting. Fitting corresponds to finding the best fit: the vector of parameters that minimizes the error between the model and the experimental data. Thus, the fitting problem is equivalent to solving an optimization problem. The optimization problem that arises from the fitting problem is a non linear optimization problem, which therefore requires a non linear optimization algorithm to be solved. A very common and powerful non linear optimization algorithm is the Levenberg-Marquardt algorithm [54]. This algorithm was used to perform the circuit fitting in this work. The Levenberg-Marquardt algorithm output is composed by the best fit parameters (the vector $\vec{a}_{R(R C)_{n}}$ that minimizes $\chi^{2}$ ); and by the standard error associated to each one of the model parameters. These standard errors quantify the uncertainty in each one of the fitted model parameters. The standard error vector, $\vec{\sigma}_{R(R C)_{n}}$, can be defined as:

$$
\vec{\sigma}_{R(R C)_{n}}=\left(\begin{array}{c}
\sigma_{R_{1}} \\
\sigma_{R R C_{1}} \\
\sigma_{C R C_{1}} \\
\vdots \\
\sigma_{R R C_{n}} \\
\sigma_{C R C_{n}}
\end{array}\right)_{2 n+1}
$$

Where $\sigma_{i}$ denotes the standard error of parameter $i$. It corresponds with a $2 n+1$ dimensional vector.

Summing up, in this work, the equivalent circuit fitting process was implemented in Labview ${ }^{\circledR}$ using a Levenberg-Marquardt algorithm coupled with an inverse-to-thesquare weighing strategy. 


\subsection{Number of subcircuits selection}

As explained in the previous subsection, the experimental spectra are fitted to a $R(R C)_{n}$ circuit. A key issue is the selection of the number of subcircuits, $n$.

The goodness of the fitting will improve by raising the number of subcircuits; however a large number of subcircuits leads to large freedom degrees for the fitting problem, and this results in very large errors in the fitted model parameters (over fitting), which makes the fitting useless for the following step of the technique. There is an optimal number of subcircuits, above which a further increase causes a greater increase in the parameter uncertainty than the obtained increase in the goodness of the fitting. The optimal number of RC subcircuits was determined individually for each spectrum.

\subsection{Montecarlo error propagation}

The equivalent circuit fitting itself is not a quantitative method: it does not allow discriminating the inconsistent points from the consistent ones; it only evaluates the consistency of the spectrum as a whole. In addition, the criterion to distinguish a "good fit" from a "bad fit" should be defined if only the equivalent circuit fitting was used. A quantitative method can be built by coupling the equivalent circuit fitting (described in previous subsections) with an error propagation method based on a Montecarlo algorithm [36].

The Montecarlo algorithm is a stochastic algorithm that simulates the random behaviour of a real system by generating pseudo-random numbers using a computer [55]. The basis of an error propagation Montecarlo algorithm is to consider the input parameters of the model as random variables with a given distribution. Using a pseudo-random number generator, a random value is generated for each input parameter, consistent with the probability distribution of that input. Using the set of random parameters generated for each input parameter, the output is calculated using the model. Repeating this process a very large number of iterations, a sample of the possible outputs of the model with the considered distributions of the input parameters is obtained. If the number of iterations is high enough, the sample estimates (sample mean and sample standard deviation) will be good estimators of the population parameters (mean and standard deviation of the model output). This information of the distribution of the model output can be used to build a consistency zone of the output variable, with a given degree of confidence.

So, in this work, once the experimental spectrum was fitted to the circuit, the fitted model parameters and their errors were used to propagate the errors through the model, using a Montecarlo algorithm. Firstly, each circuit element was assigned a 
random variable value, normally distributed around the fitted value of that model parameter and with a standard deviation equal to the fitting error for that model parameter. The normality assumption is a good hypothesis when no a priori information of the distribution is available [56]. Therefore, the random vector of input parameters of the model, $\vec{X}_{\text {ran }}$, is generated according to the following distribution:

$$
\vec{X}_{\text {ran }} \sim \mathcal{N}\left(\vec{a}_{R(R C)_{n}} ; \vec{\sigma}_{R(R C)_{n}}\right)
$$

Where $\mathcal{N}$ denotes the normal distribution. For the sake of simplicity a component-tocomponent vectorial notation has been used: the $i$-th component of vector $\vec{X}_{\text {ran }}$ is distributed according to a normal distribution of mean the $i$-th component of vector $\vec{a}_{R(R C)_{n}}$ and standard deviation the $i$-th component of vector $\vec{\sigma}_{R(R C)_{n}} \cdot \vec{a}_{R(R C)_{n}}$ is the vector of fitted values of each circuit element; and $\vec{\sigma}_{R(R C)_{n}}$ represents the fitting standard error vector. Both, $\vec{a}_{R(R C)_{n}}$ and $\vec{\sigma}_{R(R C)_{n}}$ were determined in the equivalent circuit fitting step, as described in previous subsections.

Secondly, once the random vector of input parameters of the model was generated, the random parameters were introduced in the equations of the model (4) and (5) in order to calculate the real part and the imaginary part of the impedance for every frequency in the experimental frequency range, obtaining the simulated spectrum. This corresponds to a single iteration. The model spectrum was simulated a large number of times (in the order of hundreds of thousands). Since each simulation had different model parameters (since the model parameters are random variables), the simulated spectrum differed from one simulation to the other. For each frequency of the spectrum, the sample mean and the sample standard deviation were calculated from the whole collection of simulated spectra.

On the one hand, the sample means were calculated using the following expressions:

$$
\begin{gathered}
\overline{Z^{\prime}}\left(\omega_{i}\right)=\frac{1}{n_{\text {ite }}} \cdot \sum_{k=1}^{n_{\text {ite }}} Z^{\prime}\left(\omega_{i}\right)_{k} \\
\overline{Z^{\prime \prime}}\left(\omega_{i}\right)=\frac{1}{n_{\text {ite }}} \cdot \sum_{k=1}^{n_{\text {ite }}} Z^{\prime \prime}\left(\omega_{i}\right)_{k}
\end{gathered}
$$

Where $n_{\text {ite }}$ denotes the number of iterations. $\overline{Z^{\prime}}\left(\omega_{i}\right)$ and $\overline{Z^{\prime \prime}}\left(\omega_{i}\right)$ represent the sample means of the real part and the imaginary part of the impedance for angular frequency $\omega_{i}$. 
On the other hand, the sample standard deviations were calculated using the following expressions:

$$
\begin{aligned}
s_{Z^{\prime}\left(\omega_{i}\right)} & =\sqrt{\frac{1}{n_{\text {ite }}-1} \cdot \sum_{k=1}^{n_{\text {ite }}}\left(Z^{\prime}\left(\omega_{i}\right)_{k}-\overline{Z^{\prime}}\left(\omega_{i}\right)\right)^{2}} \\
s_{Z^{\prime \prime}\left(\omega_{i}\right)}= & \sqrt{\frac{1}{n_{\text {ite }}-1} \cdot \sum_{k=1}^{n_{\text {ite }}}\left(Z^{\prime \prime}\left(\omega_{i}\right)_{k}-\overline{Z^{\prime \prime}}\left(\omega_{i}\right)\right)^{2}}
\end{aligned}
$$

Where $s_{Z^{\prime}\left(\omega_{i}\right)}$ and $s_{Z^{\prime \prime}\left(\omega_{i}\right)}$ denote the sample standard deviations of the real part and the imaginary part of the impedance for angular frequency $\omega_{i}$.

Therefore, four sample parameters (two means and two standard deviations; one associated to the real part and another to the imaginary part) are calculated for each frequency in the experimental frequency range.

As explained previously, the theoretical foundation of Montecarlo algorithm is that for a high enough number of iterations, the sample parameters tend to the parameters of the output population [55]. Therefore:

$$
\begin{aligned}
\lim _{n_{i t e} \rightarrow+\infty} \overline{Z^{\prime}}\left(\omega_{i}\right) & =\mu_{Z^{\prime}\left(\omega_{i}\right)} \\
\lim _{i t e^{\rightarrow+\infty}} \overline{Z^{\prime \prime}}\left(\omega_{i}\right) & =\mu_{Z^{\prime \prime}\left(\omega_{i}\right)} \\
\lim _{n_{i t e} \rightarrow+\infty} S_{Z^{\prime}\left(\omega_{i}\right)} & =\sigma_{Z^{\prime}\left(\omega_{i}\right)} \\
\lim _{n_{i t e} \rightarrow+\infty} S_{Z^{\prime \prime}\left(\omega_{i}\right)} & =\sigma_{Z^{\prime \prime}\left(\omega_{i}\right)}
\end{aligned}
$$

Where $\mu_{Z^{\prime}\left(\omega_{i}\right)}$ and $\sigma_{Z^{\prime}\left(\omega_{i}\right)}$ are the mean and the standard error of the real part of the impedance for angular frequency $\omega_{i}$; and $\mu_{Z^{\prime \prime}\left(\omega_{i}\right)}$ and $\sigma_{Z^{\prime \prime}\left(\omega_{i}\right)}$ are the analgoue parameters of the imaginary part of the impedance for thar angular frequency.

Therefore, if the number of iterations is high enough, then the population parameters (the parameters of interest) can be deduced from the value of the sample parameters, determined with the simulation process. A convergence study was done in order to guarantee that the number of iterations was high enough in order to obtain the population parameters from the sample estimators with a low error. 
Thirdly, the Kolmogorov-Smirnov normality test was applied in order to test the normality of each one of the output distributions (one for the real part and another one for the imaginary part, of each frequency in the experimental frequency range). In this case, the Kolmogorov-Smirnov normality test showed that there were not enough statistical evidences to reject the normality assumption for any of the output distributions. For this reason, the normality assumption was accepted for every output distribution. Therefore, for each $\omega_{i}$ in the experimental frequency range:

$$
\begin{gathered}
Z^{\prime}\left(\omega_{i}\right) \sim \mathcal{N}\left(\mu_{Z^{\prime}\left(\omega_{i}\right)} ; \sigma_{Z^{\prime}\left(\omega_{i}\right)}\right) \\
Z^{\prime \prime}\left(\omega_{i}\right) \sim \mathcal{N}\left(\mu_{Z^{\prime \prime}\left(\omega_{i}\right)} ; \sigma_{Z^{\prime \prime}\left(\omega_{i}\right)}\right)
\end{gathered}
$$

Knowing the distribution of a random variable allows to build a confidence interval that contains the random variable with a given confidence level [55].

According to the normal distribution properties [56]:

$$
\begin{gathered}
\mathbb{P}[\mu-\sigma \leq \mathcal{N}(\mu ; \sigma) \leq \mu+\sigma]=0.6826 \\
\mathbb{P}[\mu-2 \sigma \leq \mathcal{N}(\mu ; \sigma) \leq \mu+2 \sigma]=0.9545 \\
\mathbb{P}[\mu-3 \sigma \leq \mathcal{N}(\mu ; \sigma) \leq \mu+3 \sigma]=0.9973
\end{gathered}
$$

Where $\mathbb{P}[A]$ denotes the probability of event $\mathrm{A}$.

These properties were used together with the normality assumption in order to build a consistency region for each one of the output variables, with a known confidence level. The following consistency regions were defined for each frequency:

$$
\begin{gathered}
Z^{\prime}\left(\omega_{i}\right) \in\left[\mu_{Z^{\prime}\left(\omega_{i}\right)}-k \cdot \sigma_{Z^{\prime}\left(\omega_{i}\right)} ; \mu_{Z^{\prime}\left(\omega_{i}\right)}+k \cdot \sigma_{Z^{\prime}\left(\omega_{i}\right)}\right] \\
Z^{\prime \prime}\left(\omega_{i}\right) \in\left[\mu_{Z^{\prime \prime}\left(\omega_{i}\right)}-k \cdot \sigma_{Z^{\prime \prime}\left(\omega_{i}\right)} ; \mu_{Z^{\prime \prime}\left(\omega_{i}\right)}+k \cdot \sigma_{Z^{\prime \prime}\left(\omega_{i}\right)}\right]
\end{gathered}
$$

Where $k$ is a constant that depends on the selected confidence level. Table 1 gives the value of $k$ for the typical confidence levels. It also gives the probability of type I error for each confidence level: the probability to classify as inconsistent a point that is consistent.

The criteria used in this work were: on the one hand, all the experimental points inside the consistency region can be considered to satisfy the Kramers-Kronig relations (with 
a given confidence level), and therefore are consistent and can be used in the spectrum further analysis. In contrast, any point outside the consistency region does not satisfy the Kramers-Kronig relations (with a given confidence level), and consequently does not satisfy the four conditions, hence it has to be removed in order to avoid misleading conclusions from the analysis of the spectrum.

\subsection{Methodology summary}

In conclusion, the selected method for this work is divided in two parts: firstly, the experimental spectrum is fit to an equivalent circuit. Secondly, the results of the fit are used to feed a Montecarlo based error propagation method that will build an acceptance zone for each individual point of the experimental spectrum, for a given confidence level. On the one hand, experimental points inside the associated acceptance zone can be considered consistent, for that confidence level; and therefore used in further analysis of the spectrum. On the other hand, experimental points outside the associated acceptance zone can be considered inconsistent, for that confidence level; and therefore should be deleted from further analysis of the spectrum. Figure 2 sums up the overall methodology proposed in this work. 


\section{Experimental work}

The aim of the experimental part of this work is to verify that the implemented method is able to detect no stationarities. In order to achieve this goal, the impedance spectrum of an individual cell of a commercial PEM fuel cell was obtained experimentally, in stable and in unstable conditions (flooding).

The experimental setup is represented in figure 3. Its main element is a $300 \mathrm{~W}$ commercial FC stack, provided by HeliocentriS $囚$, composed by 20 individual cells, with an effective area of $58 \mathrm{~cm}^{2}$. The air supply is provided by a compressor and the hydrogen comes from a 200 bar high-pressure storage tank. The humidification of the gas inlets is assured by a humidification system and the fuel cell stack operating temperature is controlled by a refrigeration system. On the one hand, the humidification system consists in two independent bubbling humidification systems, with humidification temperature control. On the other hand, the refrigeration system consists in a heat exchanger equipped with a continuous pump and a temperature controller. The reactant gases flow rates are controlled using mass flow controllers. The reactant inlet pressures are monitored by pressure gauges and are regulated using manual valves. All the relevant system temperatures are monitored by thermocouples.

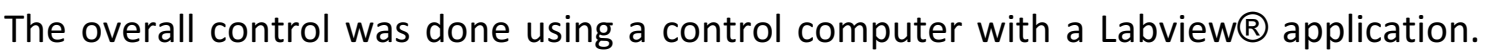
All the experiments were carried out in dead end anode mode. The individual cell galvanostatic impedance spectra were obtained using an Autolab $\circledast 302 \mathrm{~N}$ potentiostat/galvanostat with FRA module, controlled using NOVA® software.

The selected frequency range extended from $1800 \mathrm{~Hz}$ to $250 \mathrm{mHz}$, with 50 frequencies logarithmically spaced. All the spectra were obtained for a DC current of $1 \mathrm{~A}$. The perturbation amplitude was set to $5 \%$ of the DC current [10].

The impedance spectrum was measured in stable conditions, in slight flooding conditions and in severe flooding conditions. The operation parameters related to each situation are given in table 2 . It was determined in a preliminary work that each one of these set of operation parameters leads to the corresponding situation (no flooding, slight flooding or severe flooding). Each spectrum measurement was repeated 3 times, in order to obtain representative spectra.

A 15 min preconditioning was performed before each measurement in order to assure that the initial point was the same in all the experiments. The preconditioning was done in no flooding conditions. After the preconditioning, the operation conditions were set to the conditions associated with the experiment that was going to be performed; and simultaneously, the measurement process was started. In this way, a 
time drift (no stationarity) was achieved in the slight flooding and severe flooding experiments: in the second case, the time drift was of higher magnitude. 


\section{$\underline{\text { 4. Results and discussion }}$}

\subsection{Nyquist diagrams}

Figure 4 shows the Nyquist diagram of the experimental spectra obtained in the three conditions.

It can be observed that the initial points, in the high frequency zone, are the same in the three cases. This is due to the fact that the initial state was the same in the three experiments, as it was explained in the experimental setup section. The three spectra are formed by two capacitive depressed semi-circles. The detailed analysis of these spectra was done by Pérez-Page and co-workers [57-58], and is out of the scope of this work.

On the one hand, comparing the spectrum obtained in no flooding conditions with the spectrum obtained in minor flooding conditions it is observed that qualitatively they have the same shape. However, it will be shown with the application of the Montecarlo based validation method that the no flooding spectrum is a valid spectrum; whereas the minor flooding spectrum has inconsistent points with the stationarity hypothesis. Thus, two spectra with the same Nyquist shape may not be equivalent from a validity point of view: the validity of an impedance spectrum cannot be determined directly from the Nyquist diagram.

On the other hand, the spectrum obtained in severe flooding conditions presents a significant scattering in the low frequency zone. In this case, a clue of the non stationarity can be obtained directly from the Nyquist plot. However, the Nyquist plot is useless to determine which points are consistent and which ones are inconsistent.

In conclusion, Nyquist diagrams are not effective tools to determine if an experimental spectrum is valid or not; and to discriminate the consistent points from the inconsistent ones.

\subsection{Selection of the number of subcircuits and equivalent circuit fitting and}

As discussed in section 2, the first step of the proposed method is to select the number of subcircuits, $n$. In order to achieve this goal, the experimental spectra were fitted to Voight's circuit, with different number of subcircuits. Figures 5, 6 and 7 show the results obtained for the non-flooding spectrum. Similar results were obtained for the slight flooding and the severe flooding spectra. 
On the one hand, figure 5 shows the determination coefficient, $R^{2}$, of the fit as a function of the number of subcircuits. The figure shows the results for the total determination coefficient, and for the determination coefficients associated only to the real part and only to the imaginary part. All three determination coefficients show the same trend with $n$. The determination coefficient is a parameter that quantifies the goodness of the fitting: a determination coefficient of 1 corresponds to a "perfect fitting" (The model is able to simulate the $100 \%$ of the experimental variance). It is observed that initially $R^{2}$ raises with the number of subcircuits; and then it asymptotically tends to the unity. Thus, initially the consideration of a supplementary subcircuit improves significantly the fitting; but after a certain number of subcircuits, further increases of the number of subcircuits do not improve significantly the fitting. In this case, the fitting does not improve significantly by the addition of a supplementary subcircuit from $n=5$.

On the other hand, figures 6 and 7 show the evolution of the standard error associated to the different model parameters with the number of subcircuits. The standard error associated to parameter $R_{1}, \sigma_{R_{1}}$, is given by figure 6 . It is observed that initially it varies with the number of subcircuits; but after $n=4$ it remains constant.

The number of parameters $R R C_{i}$ and $C R C_{i}$ varies with $n$. Consequently, rather than representing the individual evolution of each one of these parameters, the mean standard errors are considered:

$$
\begin{aligned}
& \overline{\sigma_{R R C}}=\frac{1}{n} \cdot \sum_{i=1}^{n} \sigma_{R R C_{i}} \\
& \overline{\sigma_{C R C}}=\frac{1}{n} \cdot \sum_{i=1}^{n} \sigma_{C R C_{i}}
\end{aligned}
$$

Where $\overline{\sigma_{R R C}}$ denotes the mean standard error of parameters $R R C_{i}$; and $\overline{\sigma_{C R C}}$ is the mean standard error of parameters $C R C_{i}$. These parameters are the most natural way to combine the individual standard errors in one unique parameter for each group of parameters (resistances and capacitances).

Figure 7 shows the evolution of the mean standard errors with the number of subcircuits. It can be observed that the mean standard errors raise with the number of subcircuits: initially in a controlled way; and afterwards significantly faster. For instance, the introduction of a supplementary subcircuit for $n=5$ causes an order of magnitude change in the mean standard errors (the mean standard error associated to $C R C_{i}$ parameters is out of the figure's scale). 
In conclusion, for $n=5$ the introduction of a supplementary subcircuit does not increase significantly the goodness of the fitting (the model is able to nearly perfectly simulate the experimental spectrum); and causes a great increase of the mean standard errors. This is due to overfitting, as it was discussed in section 2 . Thus, the optimal number of subcircuits in this case is $n=5$. The same result was obtained for the slight flooding and severe flooding spectra.

The obtained fits for $n=5$ are shown in figure 4 together with the experimental data. The Montecarlo based error propagation algorithm was applied to these fits in order to obtain the consistency regions presented in section 4.4 , as it was described in section 2.

\subsection{Montecarlo algorithm convergence study}

As it was described in section 2.5, the convergence of the sample parameters to their population analogues requires a high enough number of iterations. Thus a convergence study is required in order to determine the order of magnitude of the number of iterations that guarantees the convergence.

Figures 8 and 9 represent the results of the convergence study for the minimum frequency: on the one hand, figure 8 shows the evolution of the sample means (of the real part and of the imaginary part) for the first 800 iterations; on the other hand, figure 9 shows the evolution of the sample standard deviations (of the real part and of the imaginary part) for the first 800 iterations. Analogue convergence studies were done for the rest of the frequencies in the experimental frequency list.

It can be observed that after 800 iterations the 4 studied parameters ( 2 parameters (mean and standard deviation) and 2 components (real part and imaginary part)) have converged. Thus, it can be deduced that the convergence of the Montecarlo method is quite quick: with around 1000 iterations, the sample parameters are good estimations of their population analogues. Similar results were obtained for the other frequencies. In this work, to ensure convergence, the number of performed iterations was two orders of magnitude higher: 100000 iterations were performed for each experimental spectrum. As an additional convergence verification layer, the sample parameters vs number of iterations graphs for every frequency in the experimental frequency range were observed to verify that all the sample parameters had converged. 


\subsection{Consistency zones}

Figures 10, 11 and 12 show the obtained results for non flooding, slight flooding and severe flooding conditions respectively. These figures consist in the real and imaginary impedance components versus the frequency plot obtained in each case. The experimental points are represented with the consistency region overlaid: the consistency region is formed by an upper and a lower limit. The number of simulations used in the Montecarlo algorithm was 100000; and the consistency region was built for a confidence level of $95.45 \%$. The inconsistent points are clearly identified in this kind of representation.

On the one hand, it can be observed that in no flooding conditions, all the experimental points (except an anomalous point, associated with $50 \mathrm{~Hz}$ grid coupling) are within the consistency zone. It can be deduced that the four conditions are satisfied for all these experimental points; and therefore the spectrum is valid, and can be analysed without inconsistency bias or errors risk.

On the other hand, in flooding conditions, the flooding phenomenon itself causes a non stationarity: a time drift in the PEMFC system. This time drift mainly affects the low frequency points of the spectrum, since they are the most time consuming ones. It can be observed that the presented method is able to detect the time drift, both in light and in severe flooding conditions. The advantage of the Montecarlo method is that it discriminates between the experimental points for which the time drift is significant and the experimental points that due to the little measurement time (high frequencies) are not significantly affected by the time drift. Therefore, the inconsistent points can be deleted from the spectrum; and the refined spectrum can be analysed with the certainty that no bias or errors are being introduced in the analysis because of the non stationarity of the system.

These results show the power of the implemented validation technique: it can even detect no stationarities that do not affect the shape of the impedance spectrum, as in the case of the minor flooding conditions. The results also show the usefulness of the implemented technique: if not used, the minor flooding spectrum would have been accepted (since there is nothing in the Nyquist plot that alerts that the spectrum contains inconsistent points); and biased conclusions would have been obtained. The validation technique does not only allow to determine if the spectrum is valid or not as a whole; but it also allows to discriminate the consistent points from the inconsistent ones. 


\section{Conclusions}

In conclusion, the equivalent electrical circuit fitting coupled with Montecarlo error propagation method manages to validate the experimental impedance spectra by building a consistency region that allows distinguishing the consistent points of an experimental spectrum from the inconsistent ones. The inconsistent points can be deleted in order to analyze the rest of the spectrum, being certain that the used data verify the conditions of linearity and stability, and therefore that the obtained conclusions are nor misleading due to the presence of time drifts or nonlinearities. 


\section{Nomenclature}

\section{Normal letters}

C Capacitance $(F)$

$n \quad$ Number of RC subcircuits in Voight circuit

$n_{\text {ite }} \quad$ Number of iterations

$N_{p} \quad$ Number of points in the impedance spectrum

$R \quad$ Resistance $(\Omega)$

$S \quad$ Sample standard deviation

$\vec{X}_{\text {ran }} \quad$ Random vector of input parameters

$\bar{X} \quad$ Sample mean

$Z \quad$ Complex impedance $(\Omega)$

$Z^{\prime} \quad$ Impedance real part $(\Omega)$

$Z^{\prime \prime} \quad$ Impedance imaginary part $(\Omega)$

\section{Greek letters}

$\begin{array}{ll}\alpha & \text { Real part weight factor } \\ \beta & \text { Imaginary part weight factor } \\ \sigma & \text { Population standard deviation } \\ \mu & \text { Population mean } \\ \chi^{2} & \text { Sum of weighed squared residuals of the model }\left(\Omega^{2}\right) \\ \omega & \text { Angular frequency }\left(\mathrm{rad} \cdot \mathrm{s}^{-1}\right)\end{array}$

\section{Subscripts}

exp Experimental spectrum points

$R C_{i} \quad \mathrm{i}$-th $\mathrm{RC}$ subcircuit of Voight circuit

$R(R C)_{n} \quad$ Voight circuit with $n$ RC subcircuits

$R(R C)_{n} C L \quad$ Boukamp circuit with $n$ RC subcircuits 


\section{Acknowledgments}

The authors are very grateful to the Generalitat Valenciana for its economic support in form of Vali+d grant (Ref: ACIF-2013-268). 


\section{References}

[1] Danzer MA, Hofer E. Electrochemical parameter identification. An efficient method for fuel cell impedance characterisation. J Power Sources 2008; 183: 55-61.

[2] Wu J, et al. Diagnostic tools in PEM fuel cell research: Part I Electrochemical techniques. Int J Hydrogen Energ 2008; 33: 1735-46.

[3] Brunetto C, Tina G, Sqadrito G, Moschetto A. PEMFC diagnosis and modeling by electrochemical impedamce spectroscopy. Proceedings of the 12th IEEE Mediterranean electrochemical conference 2004; Piscataway, NJ: 1045-50.

[4] Li G, Pickup PG. Ionic conductivity of PEMFC electrodes. J Electrochem Soc 2003; 150: C745-52.

[5] Yan X, et al. AC impedamce characteristics of a $2 \mathrm{~kW}$ PEM fuel cell stack under different operating conditions and load changes. Int J Hydrogen Energ 2007; 32: 435864.

[6] $\mathrm{Li} \mathrm{H}$, et al. A review of water flooding issues in the proton exchange membrane fuel cell. J Power Sources 2008; 178: 103-17.

[7] Nguyen TV, White RE. A water and Heat Management Model for Proton Exchange Membrane Fuel Cells. J Electrochem Soc 1993; 140: 2178-86.

[8] Song JM, Cha SY, Lee WM. Preparation and characterization of high density spherical LiNi08C002O2 cathode material for lithium secondary batteries. J Power Sources 2001; 94: 78-84.

[9] Antolini E, Giorgi L, Pozio A, Passalacqua E. Influence of Nafion loading in the catalyst layer of gas-diffusion electrodes for PEFC. J Power Sources 1999; 77: 136-42.

[10] Gode P, Jaouen F, Lindbergh G, Lundblad A, Sundholm G. Influence of the composition on the structure and electrochemical characteristics of the PEFC cathode. Electrochim Acta 2003; 48: 4175-87.

[11] Abaoud HA, Gihouse M, Lovell KV, Al-Motairy GN. Alternative formulation for proton exchange membrane fuel cell (PEMFC) electrode preparation. J New Mat Elect Syst 2003; 6: 149-55.

[12] Ciureanu M, Mikhailenko SD, Kaliaguine S. PEM fuel cells as membrane reactors: kinetic analysis by impedance spectroscopy. Catal Today 2003; 82: 195-206.

[13] Hombradosa AG, et al. Symmetrical electrode mode for PEMFC characterisation using impedance spectroscopy. J Power Sources 2005; 151: 25-31.

[14] Cooper KR, Smith M. Electrical test methods for on-line fuel cell ohmic resistance measurement. J Power Sources 2006; 160: 1088-95.

[15] Abe T, Shima H, Watanabe K, Ito Y. Study of PEFCs by AC impedance current interrupt, and dew point measurements. J Electrochem Soc 2004; 151: A101-5.

[16] Young HC, Yong GS, Seong CC, Won IW, Hak SH. Evaluation of the Nafion effect on the activity of Pt-Ru electrocatalysts for the electrooxidation of methanol. J Power Sources 2003; 118: 334-41. 
[17] Freire TJP, Gonzalez ER. Effect of membrane characteristics and humidification conditions on the impedance response of polymer electrolyte fuel cells. J Electroanal Chem 2001; 503: 57-68.

[18] Hakenjos A, Zobel M, Clausnitzer J, Habling C. Simultaneous electrochemical impedance spectroscopy of single cells in a PEM fuel cell stack. J Power Sources 2006; 154: 360-3.

[19] Yuan X, et al. AC impedance diagnosis of a 500W PEM fuel cell stack. Part I: stack impedance. J Power Sources 2006; 161: 920-8.

[20] Yuan X, Sun JC, Wang H, and Zhang J. AC impedance diagnosis of a 500W PEM fuel cell stack. Part II: Individual cell impedance. J Power Sources 2006; 161: 929-37.

[21] Cleghorn SJC, et al. A polymer electrolyte fuel cell life test: 3 years of continuous operation. J Power Sources 2006; 158: 446-54.

[22] Oszcipok M, Riemann D, Kronenwett U, Kreideweis M, Zedda M. Statistic analysis of operational influences on the cold start behaviour of PEM fuel cells. J Power Sources 2005; 145: 407-15.

[23] Mommaa A, Kagaa Y. AC impedance behavior of a practical-size single-cell SOFC under DC current. Solid State Ionics 2004; 174: 87-95.

[24] Amphlett JC, Peppley BA, Halliop E, Sadiq A. The effect of anode flow characteristics and temperature on the performance of a direct methanol fuel cell. $J$ Power Sources 2001; 96: 204-13.

[25] Springer TE, Zawodzinski TA, Wilson MS, Gottesfeld S. Characterization of polymer electrolyte fuel cells using AC impedance spectroscopy. J Electrochem Soc 1996; 143: 587-99.

[26] Barsoukov E, Macdonald JR. Impedance spectroscopy. Theory, experiment and applications. 2nd ed. New Jersey: John Wiley \& Sons, 2005.

[27] Macdonald DD, Sikora E. Characterizing electrochemical systems in the frequency domain. Electrochim Acta 1997; 43: 87-107.

[28] Orazem ME, Tribollet B. Electrochemical impedance spectroscopy. New Jersey: John Wiley \& Sons, 2008.

[29] Victoria SN, Ramanathan S. Effect of potential drift and ac amplitude on the electrochemical impedance spectra. Electrochim Acta 2011; 56: 2606-15.

[30] Hirschorn B, Tribollet B, Orazem ME. On selection of the perturbation amplitude required to avoid nonlinear effects in impedance measurements. Israel J Chem 2008; 48: 133-42.

[31] Polo JL, Torres CL, Cano E, Bastidas JM. An impedance study on corrosion of ISI 316L stainless steel in the passive and pitting regions. Rev Metal Madrid 1999; 35: 36878.

[32] Sunil KR, Hagelin-Weaver H, Orazem ME. Application of complementary analytical tools to support interpretation of polymer-electrolyte-membrane fuel cell impedance data. J Power Sources 2011; 196: 3736-42. 
[33] Kramers HA. Die dispersion und adsorption von Röntgen-stralen. Physikalische Zeitschrift 1929; 30: 522-3.

[34] Kronig RL. On the theory of dispersion of X-rays. J Opt Soc Am 1926; 12: 547-57.

[35] Bode HW. Network analysis and feedback amplifier design. New York: Van Nostrand Co Inc, 1945.

[36] Agarwal P, Orazem ME. Application of measurement models to impedance spectroscopy. III. Evaluation of consistency with the Kramers-Kronig relations. J Electrochem Soc 1995; 142: 4159-68.

[37] Urquidi-Macdonald M, Real S, Macdonald DD. Applications of Kramers-Kronig transforms in the analysis of electrochemical impedance data- III. Stability and linearity. Electrochim Acta 1990; 35: 1559-66.

[38] Hirschorn B, Orazem ME. On the sensitivity of the Kramers-Kronig relations to nonlinear effects in impedance measurements. J Electrochem Soc 2009; 156: C354-61.

[39] Orazem ME, Esteban JM, Moghissi OC. Practical applications of the KramersKronig relations. Corrosion 1991; 47: 248-59.

[40] Esteban JM, Orazem ME. On the application of the Kramers-Kronig relations to evaluate the consistency of electrochemical impedance data. J Electrochem Soc 1991; 138: 67-76.

[41] Kendig M, Mansfeld F. Technical note: Corrosion rates from impedance measurements: An improved approach for rapid automatic analysis. Corrosion 1983; 39: 466-67.

[42] Mansfeld F, Kendig M, Lorenz WJ. Corrosion inhibition in neutral, aerated media. Electrochemical science and technology. Technical reports. J Electrochem Soc 1985; 132: 290-6.

[43] Macdonald DD, Urquidi-Macdonald M. Application of Kramers-Kronig transforms in the analysis of electrochemical systems: I. Polarization Resistance. J Electrochem Soc 1985; 132: 2316-19.

[44] Haili CC. The corrosion of iron rotating hemishperes in $1 \mathrm{M}$ sulfuric acid: an electrochemical impedance study. Msc. Thesis. University of California, Berkeley; 1987. [45] Darowicki K. Frequency dispersion of harmonic components of the current of an electrode process. J Electroanal Chem 1995; 394: 81-6.

[46] Darowicki K. Theoretical description of fundamental-harmonic impedance of a two step electrode reaction. Electrochim Acta 1995; 40: 767-74.

[47] Macdonald JR, Potter LD. A flexible procedure for analysing impedance spectroscopy results: Description and illustrations. Solid State Ionics 1987; 24: 61-79.

[48] Boukamp BA. A linear Kronig-Kramers transform test for inmittance data validation. J Electrochem Soc 1995; 142: 1885-94.

[49] Bastidas DM, Cano E, López-Caballero JA, Polo JL, Bastidas JM. Application of Kramers-Kronig relationships for titanium impedance data validation in a Ringer's solution. Rev Metal Madrid 2004; 40: 303-11. 
[50] Boukamp BA. A nonlinear least square fit procedure for analysis of inmittance data of electrochemical systems. Soild State lonics 1986; 20: 31-44.

[51] Yuan XZ, Song C, Wang H, Zhang J. Electrochemical impedance spectroscopy in PEM fuel cells. Fundamentals and applications. 1st ed. London: Springer; 2010.

[52] Bevington PR, Robinson DK. Data reduction and error analysis. 3rd ed. New York: McGraw-Hill; 2003.

[53] Macdonald JR, Garber JA. Analysis of impedance and admittance data for solids and liquids. J Electrochem Soc 1977; 124: 1022-30.

[54] Nocedal J, Wright S. Numerical optimization. 2nd ed. New York: Springer; 2006.

[55] Tanner MA. Tools for statistical inference: Methods for the exploration of posterior distributions and likelihood functions. 3rd ed., New York: Springer Series in Statistics; 1996.

[56] Mendenhall W, Sincich T. Statistics for engineering and the sciences. 5th ed. London: Pearson Education; 2007.

[57] Pérez-Herranz V, Pérez-Page M. Effect of the operation and humidification temperatures on the performance of a PEM fuel cell stack on dead-end mode. Int J Electro Sci 2011; 6: 492-505.

[58] Pérez-Page M, Pérez-Herranz V. Study of the electrochemical behaviour of a 300 W PEM fuel cell stack by Electrochemical Impedance Spectroscopy. Int J Hydrogen Energ 2014; 39: 4009-15. 


\section{LIST OF TABLES.}

Table 1. Consistency region definition

Table 2. Operation parameters 


\section{LIST OF FIGURES.}

Figure 1. Voight's circuit

Figure 2. Equivalent circuit fitting coupled with Montecarlo error propagation method for construction of consistency regions

Figure 3. Experimental setup

Figure 4. Nyquist diagram

Figure 5. Determination coefficient of the fit of the no flooding spectrum to Voight's circuit with $\boldsymbol{n}$ subcircuits

Figure 6. Standard error of resistance $\boldsymbol{R}_{\mathbf{1}}$ of the fit of the no flooding spectrum to Voight's circuit with $\boldsymbol{n}$ subcircuits

Figure 7. Mean standard error of resistances $\boldsymbol{R} \boldsymbol{R} \boldsymbol{C}_{\boldsymbol{i}}$ and capacitances $\boldsymbol{C} \boldsymbol{R} \boldsymbol{C}_{\boldsymbol{i}}$ of the fit of the no flooding spectrum to Voight's circuit with $\mathrm{n}$ subcircuits

Figure 8. Real part (a) and imaginary part (b) mean convergence for minimum frequency

Figure 9. Real part (a) and imaginary part (b) standard deviation convergence for minimum frequency

Figure 10. Experimental impedance spectrum (real part (a) and imaginary part (b)) obtained for no flooding conditions (stable conditions), and the built consistency region

Figure 11. Experimental impedance spectrum (real part (a) and imaginary part (b)) obtained for slight flooding conditions, and the built consistency region

Figure 12. Experimental impedance spectrum (real part (a) and imaginary part (b)) obtained for severe flooding conditions, and the built consistency region 
Table 1. Consistency region definition

\begin{tabular}{|c|c|c|}
\hline Conf idence level (\%) & $\boldsymbol{k}$ & $\mathbb{P}[$ Error I] \\
\hline $\mathbf{6 8 . 2 6}$ & 1 & 0.3174 \\
\hline $\mathbf{9 5 . 4 5}$ & 2 & 0.0455 \\
\hline $\mathbf{9 9 . 7 3}$ & 3 & 0.0027 \\
\hline
\end{tabular}


Table 2. Operation parameters

\begin{tabular}{|c|c|c|c|}
\hline Operation parameter & $\begin{array}{c}\text { No } \\
\text { flooding }\end{array}$ & $\begin{array}{c}\text { Slight } \\
\text { flooding }\end{array}$ & $\begin{array}{c}\text { Severe } \\
\text { flooding }\end{array}$ \\
\hline $\begin{array}{c}\text { Operation } \\
\text { temperature }\end{array}$ & $30^{\circ} \mathrm{C}$ & $30^{\circ} \mathrm{C}$ & $30^{\circ} \mathrm{C}$ \\
\hline $\begin{array}{c}\text { Hydrogen } \\
\text { humidification } \\
\text { temperature }\end{array}$ & $30^{\circ} \mathrm{C}$ & $50^{\circ} \mathrm{C}$ & $70^{\circ} \mathrm{C}$ \\
\hline $\begin{array}{c}\text { Air humidification } \\
\text { temperature }\end{array}$ & $30^{\circ} \mathrm{C}$ & $50^{\circ} \mathrm{C}$ & $70^{\circ} \mathrm{C}$ \\
\hline
\end{tabular}




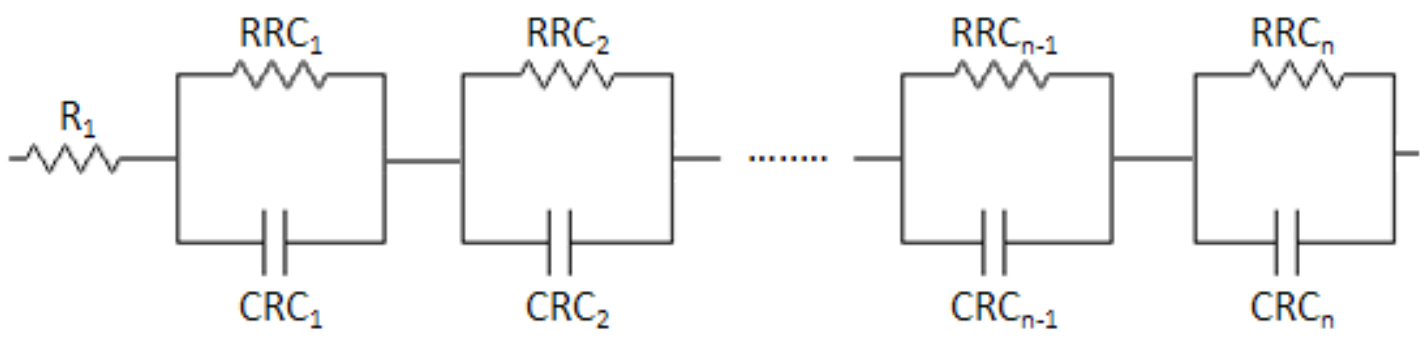

Figure 1. Voight's circuit 


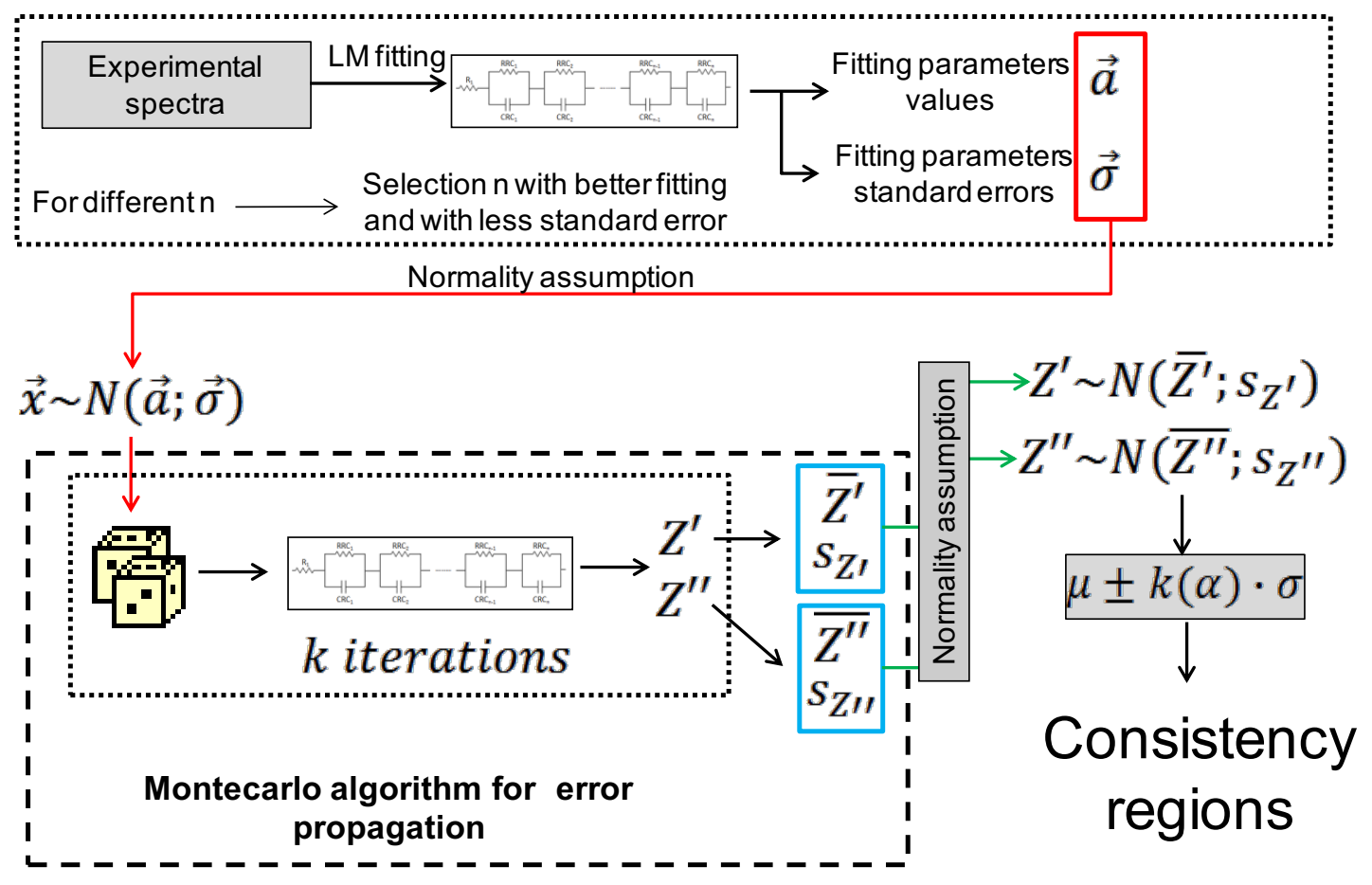

Figure 2. Equivalent circuit fitting coupled with Montecarlo error propagation method for construction of consistency regions 


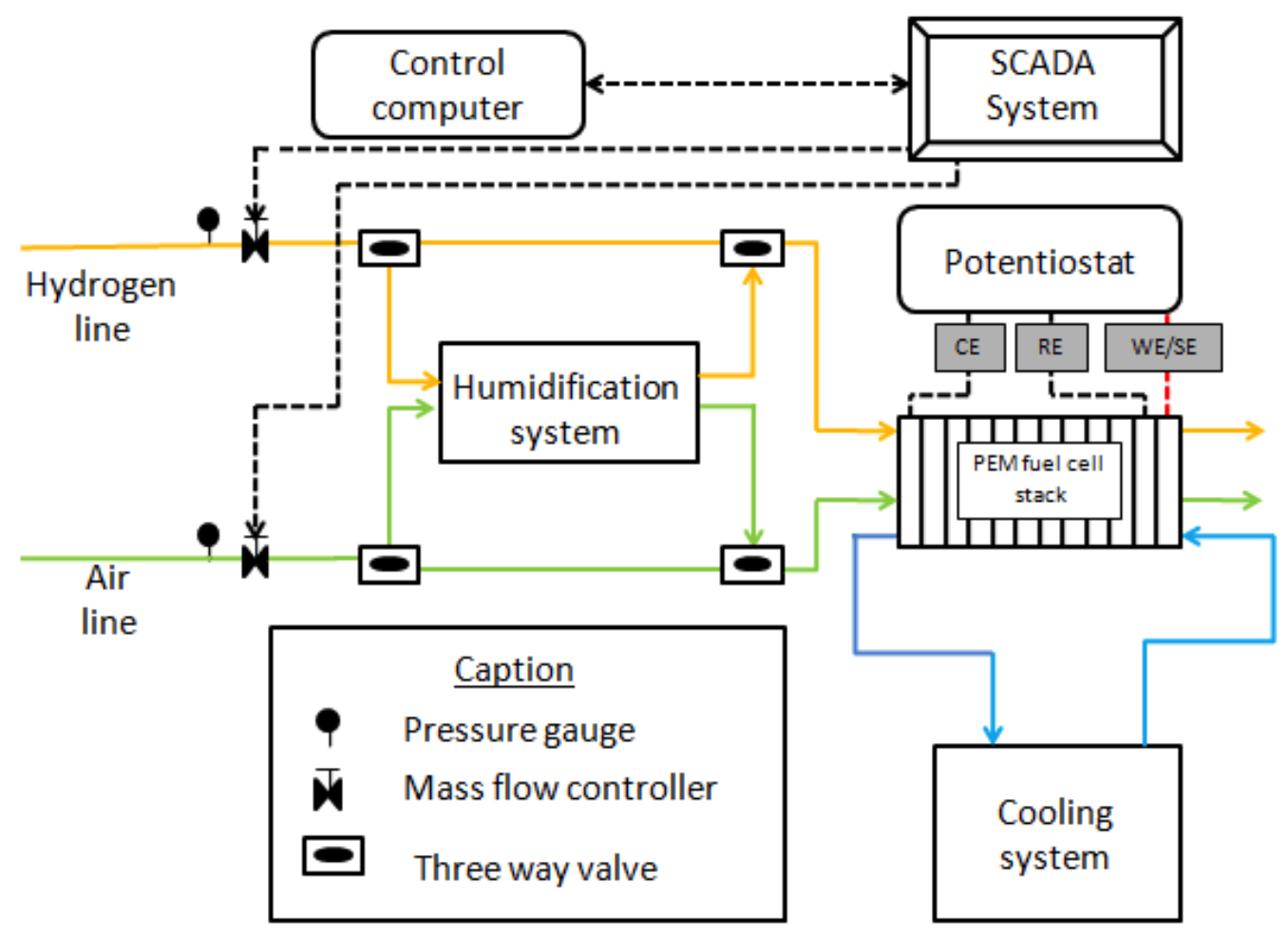

Figure 3. Experimental setup 


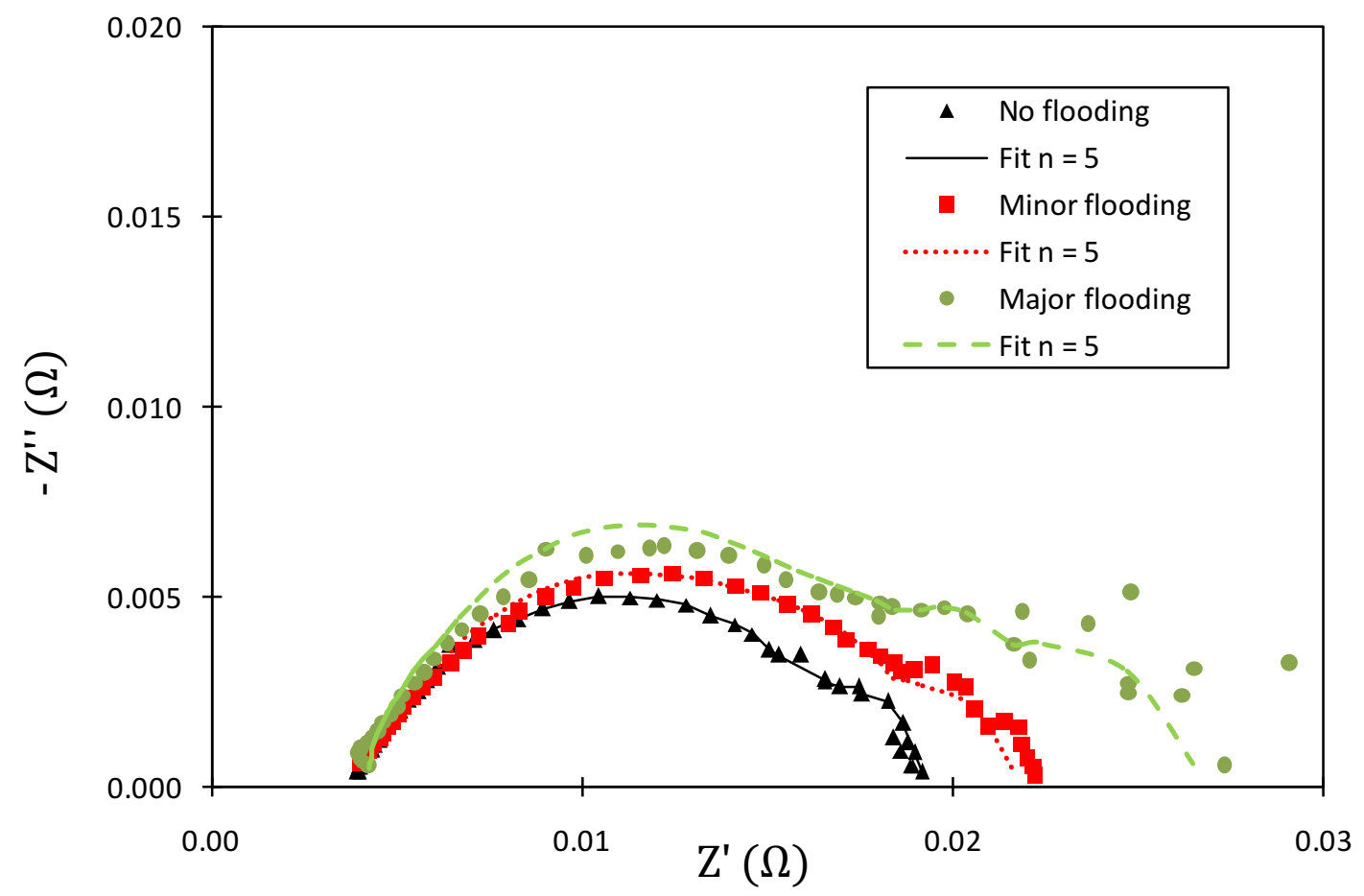

Figure 4. Nyquist diagram 


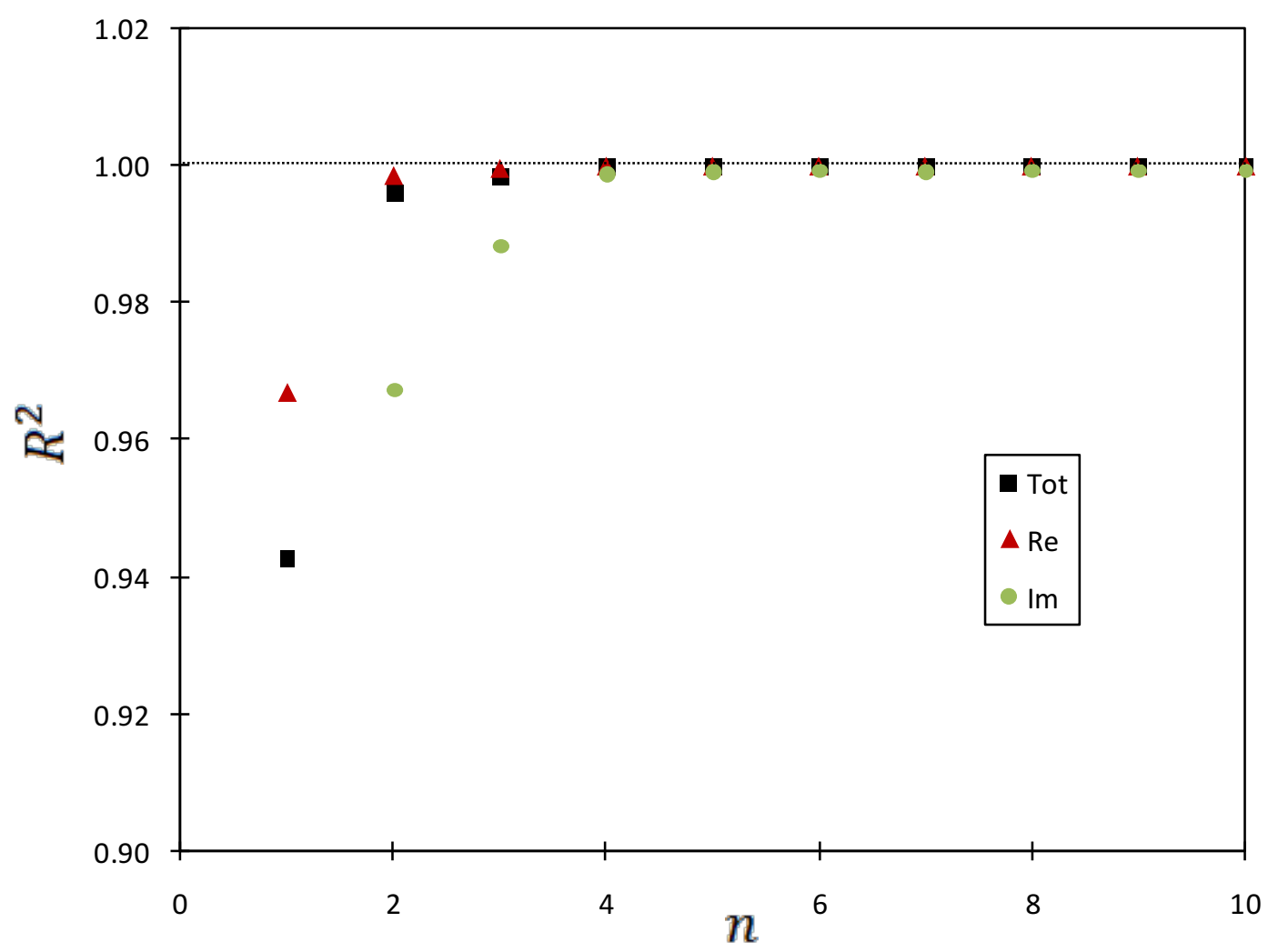

Figure 5. Determination coefficient of the fit of the no flooding spectrum to Voight's circuit with $\boldsymbol{n}$ subcircuits 


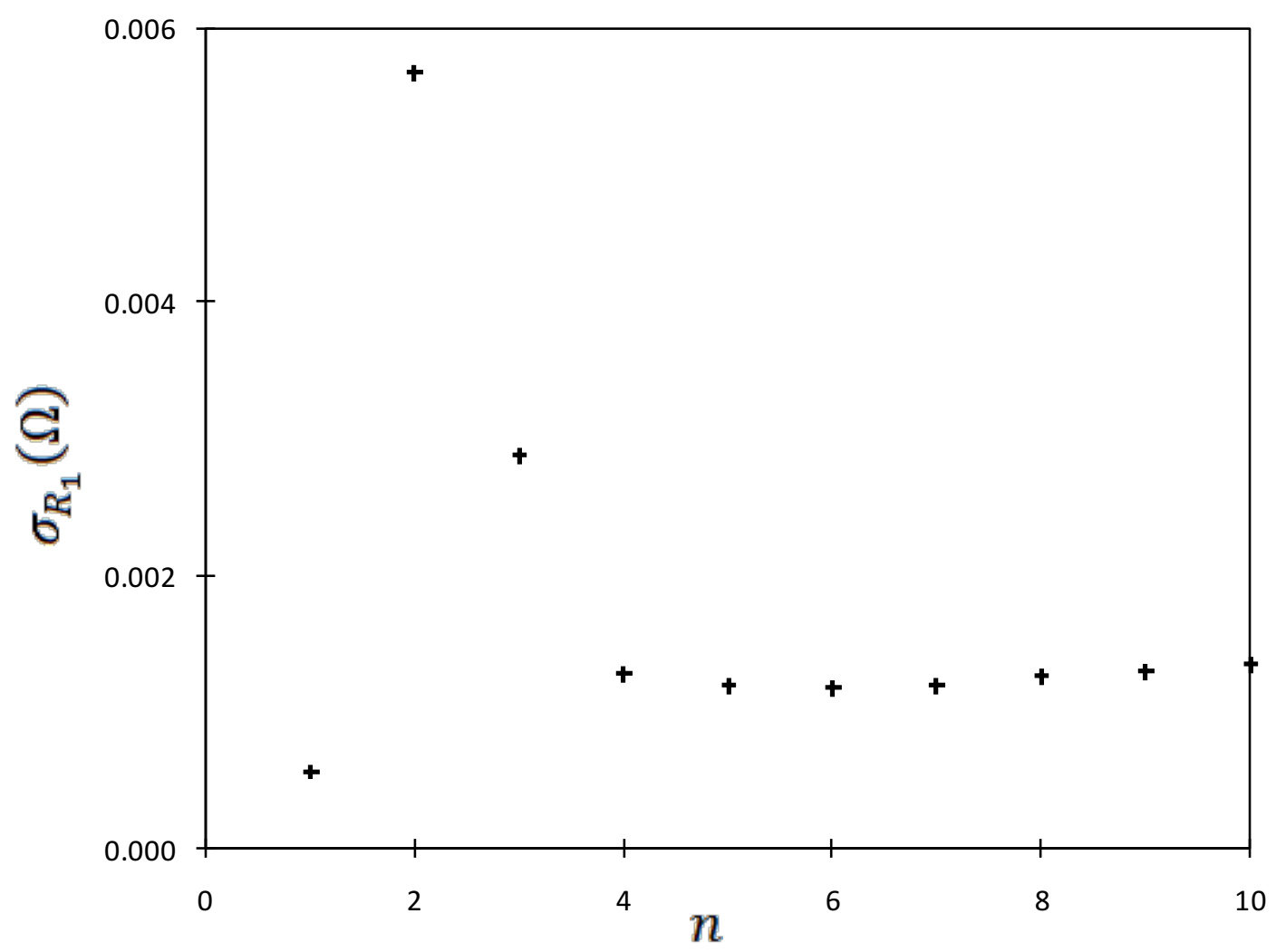

Figure 6. Standard error of resistance $\boldsymbol{R}_{\mathbf{1}}$ of the fit of the no flooding spectrum to Voight's circuit with $\boldsymbol{n}$ subcircuits 


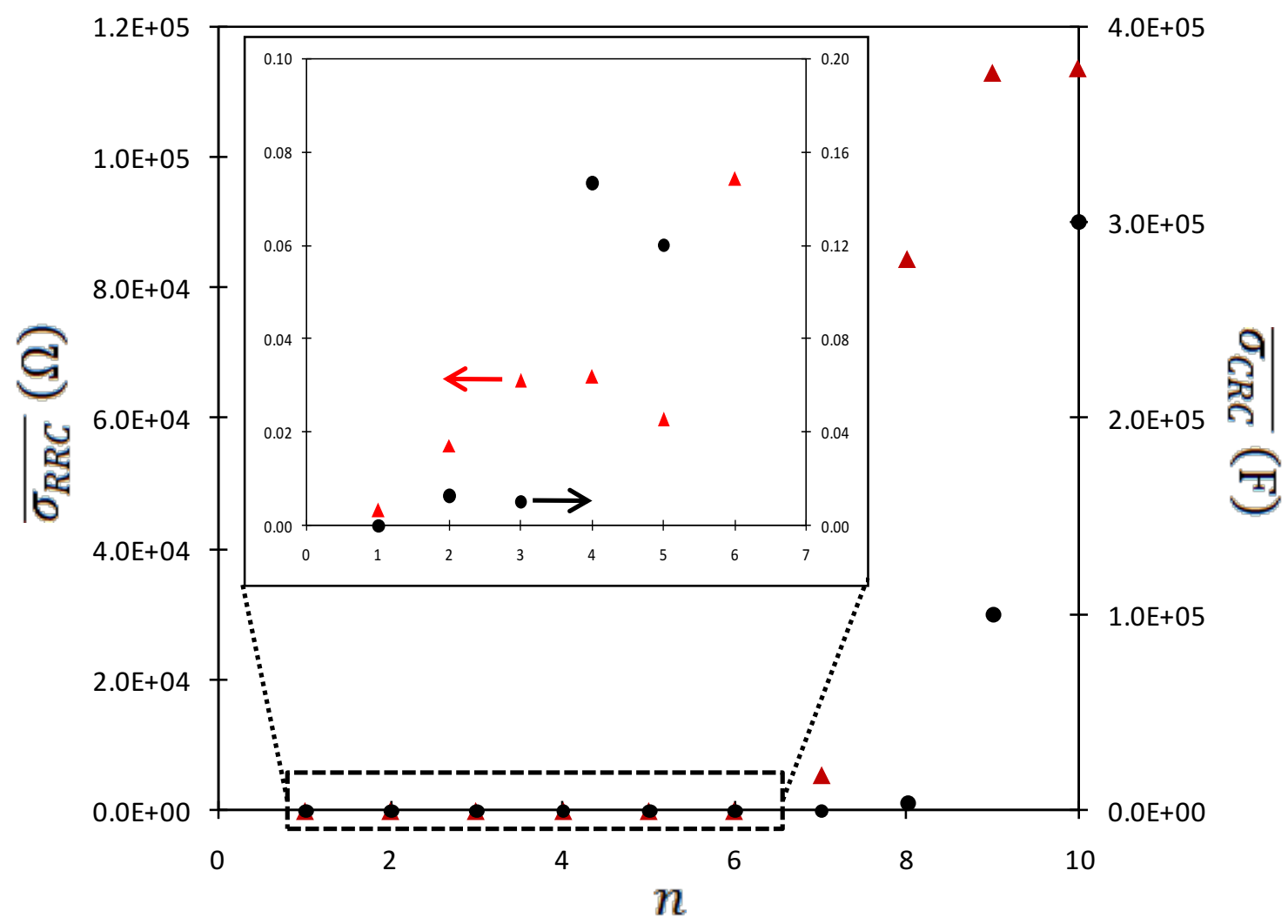

Figure 7. Mean standard error of resistances $\boldsymbol{R} \boldsymbol{R} \boldsymbol{C}_{\boldsymbol{i}}$ and capacitances $\boldsymbol{C} \boldsymbol{R} \boldsymbol{C}_{\boldsymbol{i}}$ of the fit the no flooding spectrum to Voight's circuit with $\mathrm{n}$ subcircuits 

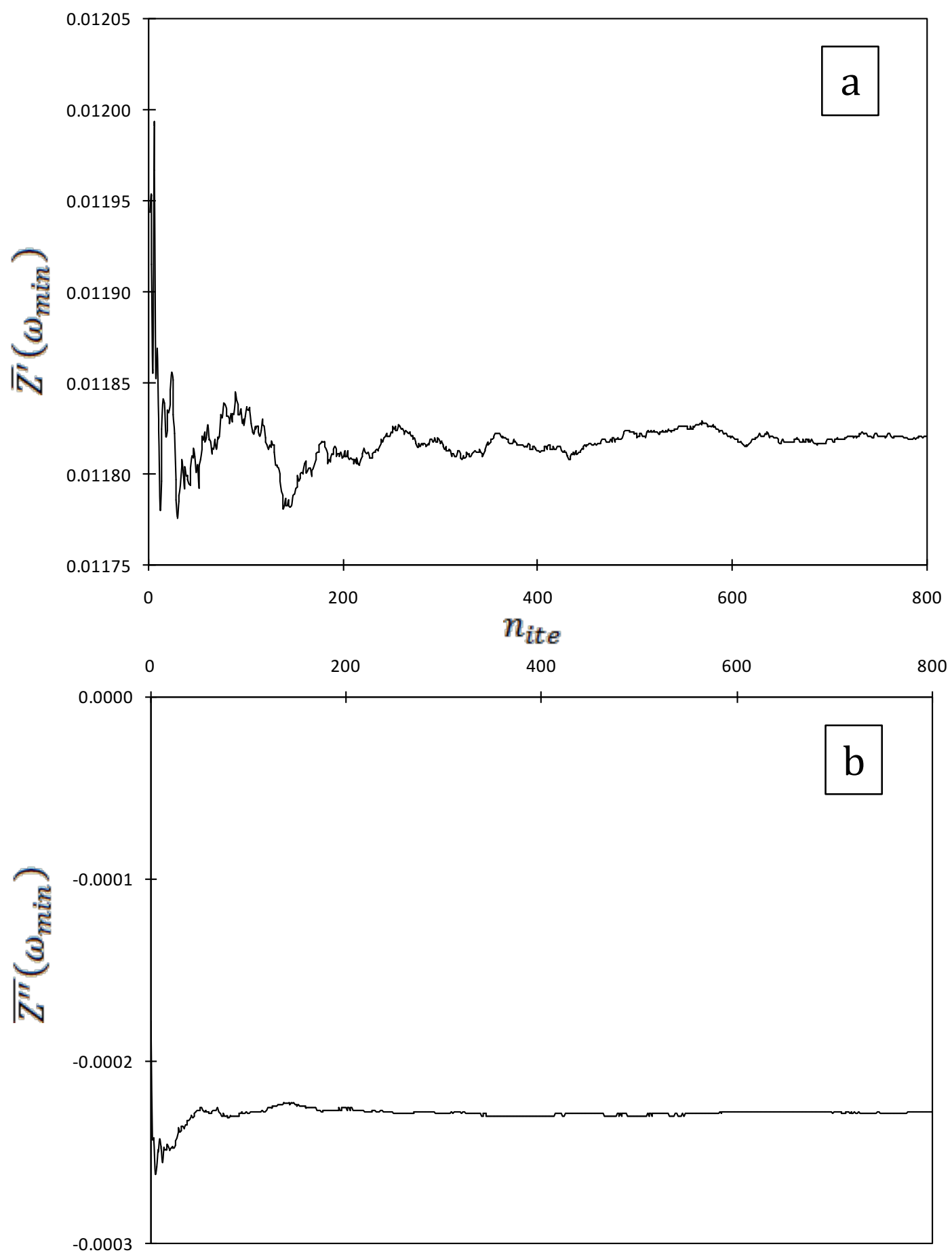

Figure 8. Real part (a) and imaginary part (b) mean convergence for minimum frequency 

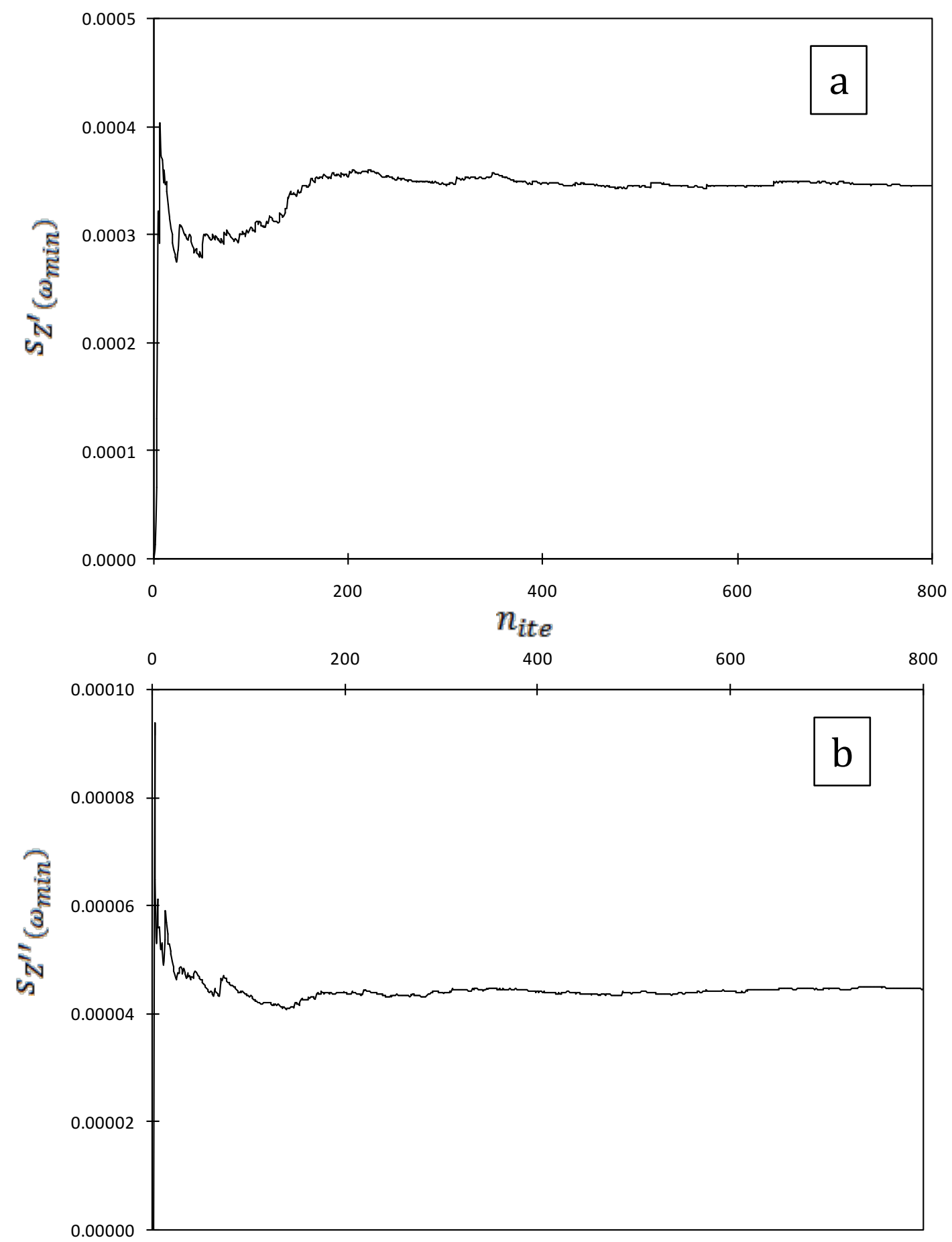

Figure 9. Real part (a) and imaginary part (b) standard deviation convergence for minimum frequency 


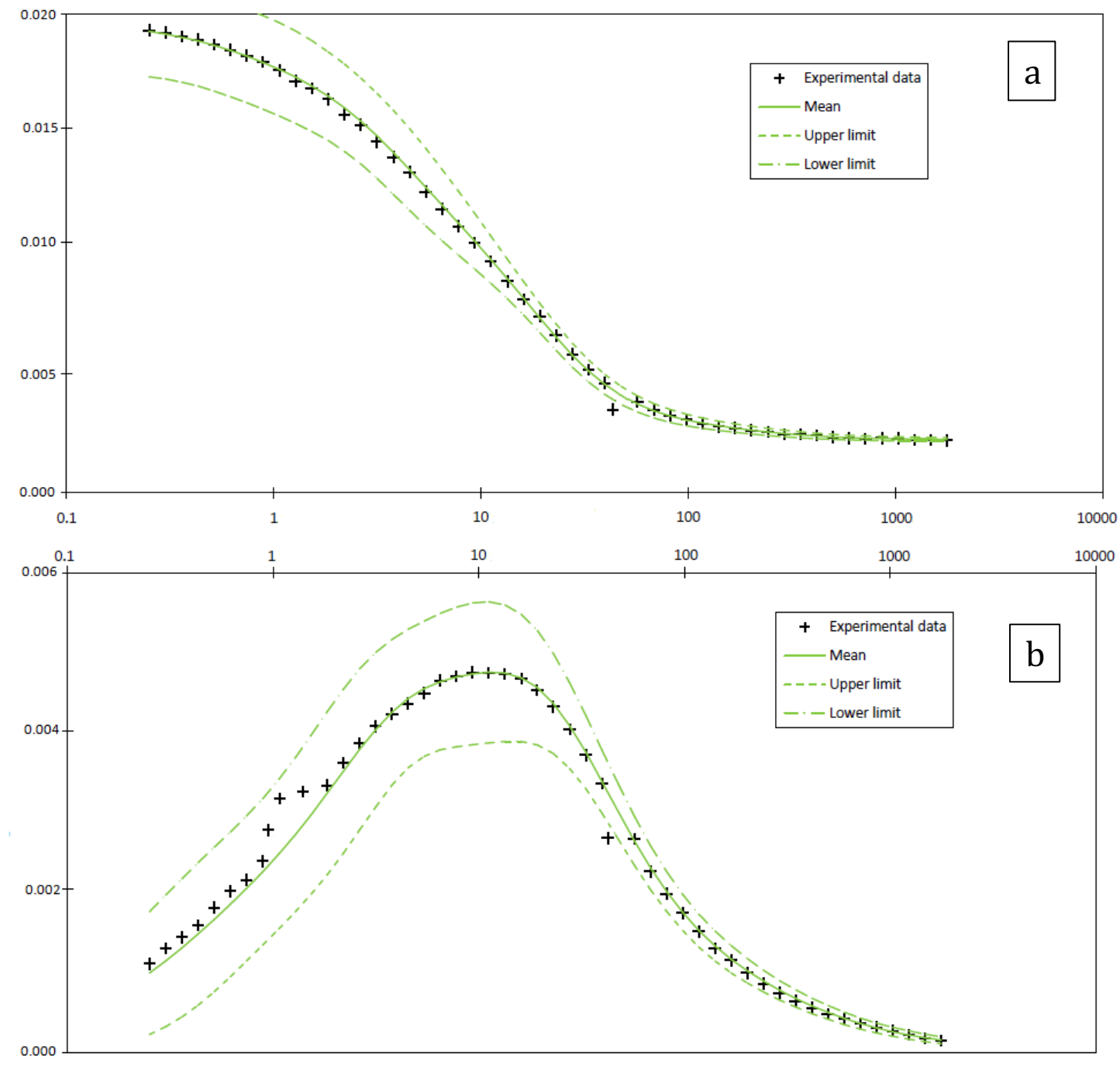

Figure 10. Experimental impedance spectrum (real part (a) and imaginary part (b)) obtained for no flooding conditions (stable conditions), and the built consistency region 

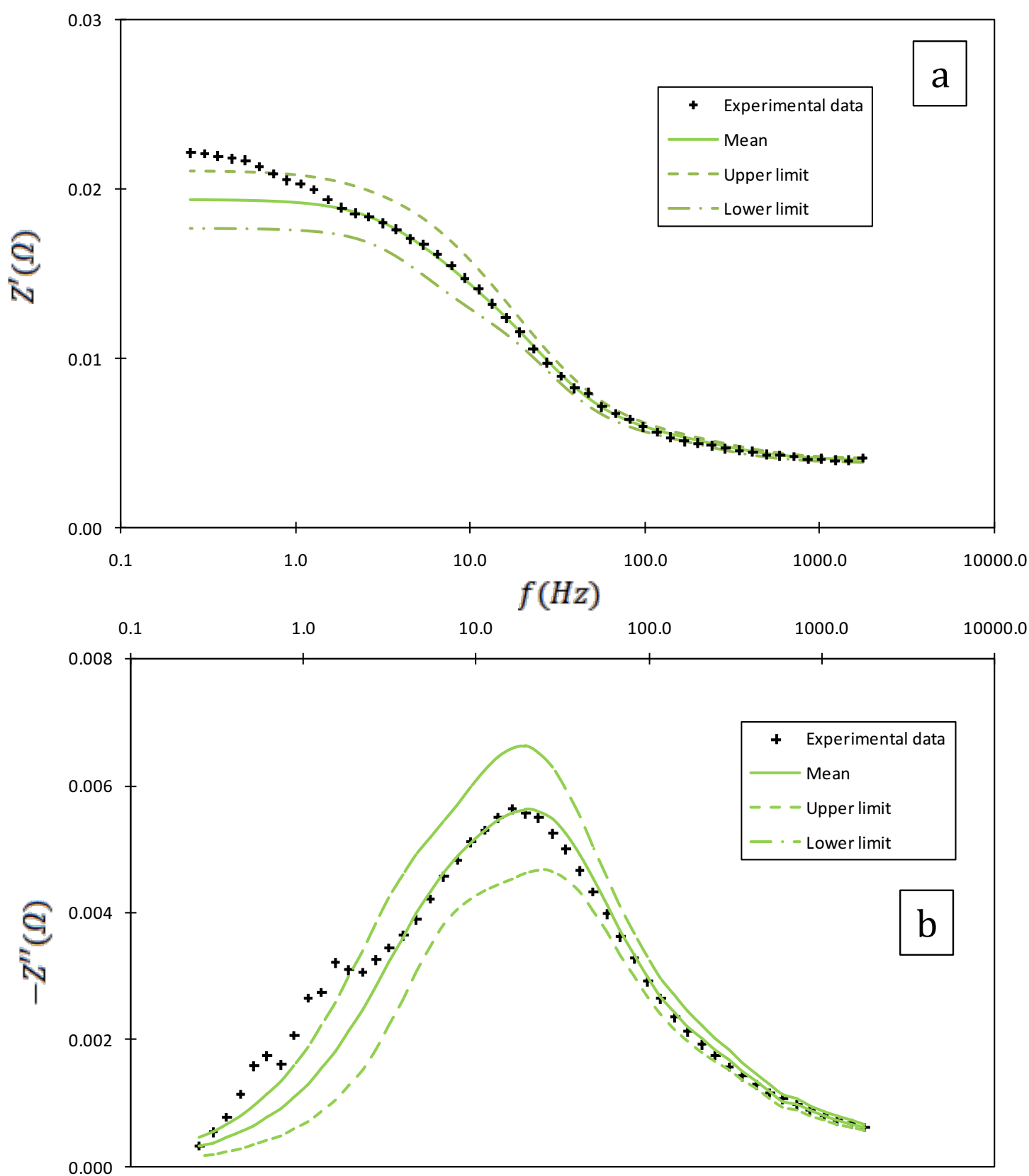

Figure 11. Experimental impedance spectrum (real part (a) and imaginary part (b)) obtained for slight flooding conditions, and the built consistency region 

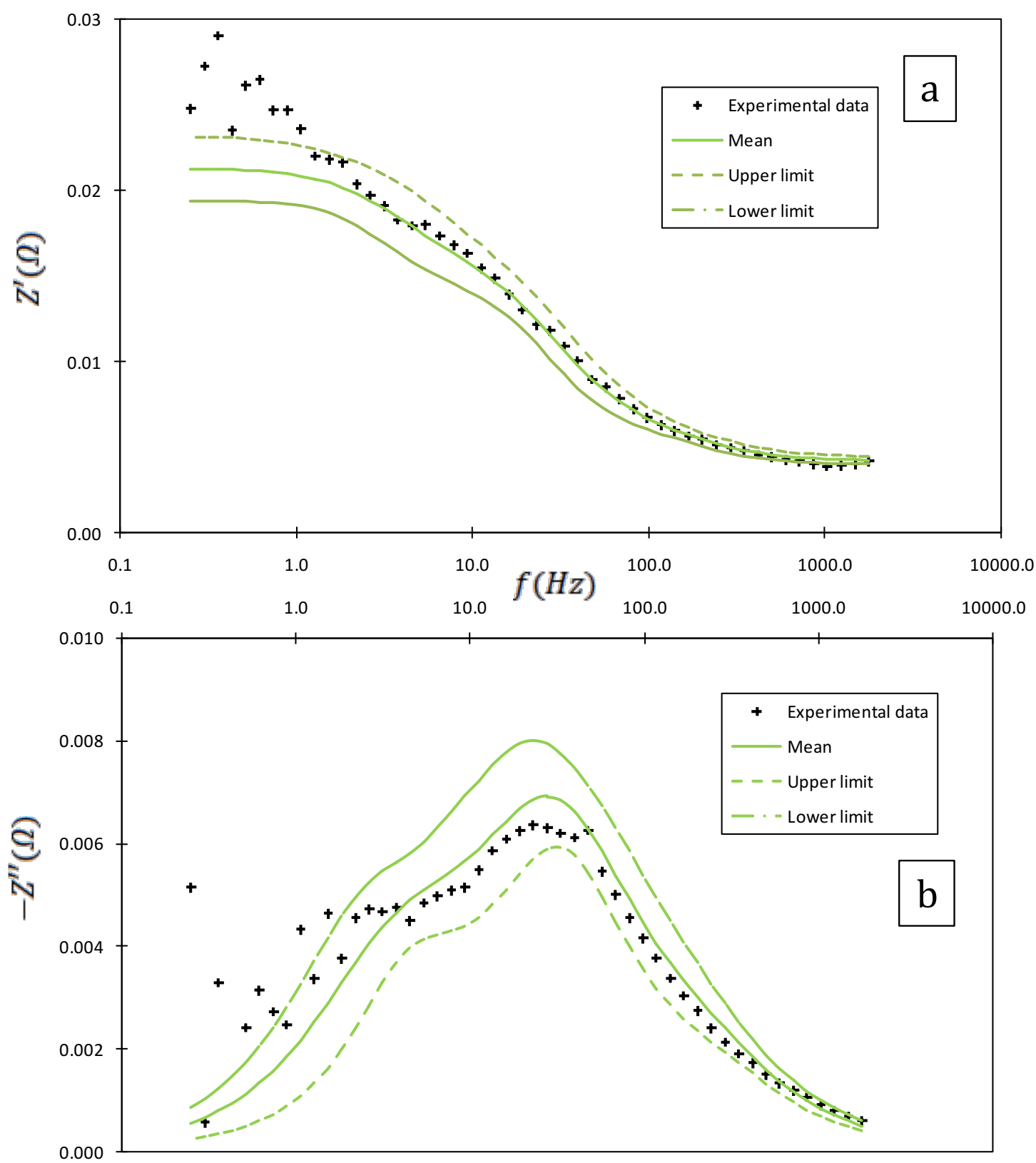

Figure 12. Experimental impedance spectrum (real part (a) and imaginary part (b)) obtained for severe flooding conditions, and the built consistency region 